\title{
Buscando determinantes de la seguridad ontológica en la España de la crisis
}

\author{
Javier CALLEJO \\ TRANSOC - Universidad Nacional de Educación a Distancia \\ mcallejo@poli.uned.es
}

Recibido: 09-06-2015

Aceptado: 08-02-2016

\begin{abstract}
Resumen
El concepto de seguridad ontológica tiene un eco notable en la sociología actual para describir el estado emocional del hombre de la modernidad tardía. Sin embargo, el concepto creado por Giddens en los años ochenta se ha utilizado poco en la investigación empírica preocupada por cubrir diversas fuentes de riesgo o incertidumbre. En este trabajo, se propone una escala de seguridad ontológica a partir de tal articulación de fuentes de riesgo. Para ello, partimos de los resultados de una investigación centrada en la relación entre riesgo, incertidumbre y vulnerabilidad en el contexto de la crisis económica en España. Estos resultados se producen a partir de nueve grupos de discusión y una encuesta con cuestionario estandarizado aplicado telefónicamente a una muestra nacional de 2.408 personas mayores de 18 años.

El trabajo se divide en tres campos principales. En el primero, se construye la escala a partir de los resultados de la aplicación de diferentes ítems presentes en el cuestionario utilizado. La segunda parte explora las relaciones de la escala obtenida con las variables más próximas a las dimensiones emocionales de los individuos. La tercera parte observa las variables que contribuyen a los cambios en la escala. Estas variables muestran la característica estructural de la seguridad ontológica.
\end{abstract}

Palabras clave: emociones; escala; confianza; modernidad tardía; seguridad.

\section{Looking for Determinants of Ontological Security in the Spain of the Crisis}

\begin{abstract}
The concept of ontological security has a remarkable echo in the current sociology to describe emotional status of men of late modernity. However, the concept created by Giddens in the eighties has been little used in empirical research covering various sources of risk or uncertainty. In this paper, a scale for ontological security is proposed. To do this, we start from the results of a research focused on the relationship between risk, uncertainty and vulnerability in the context of the economic crisis in Spain. These results were produced through nine focus groups and a telephone survey with standardized questionnaire applied to a national sample of 2,408 individuals over 18 years.

This work is divided into three main sections. In the first, a scale has been built from the results of the application of different items present in the questionnaire used. The second part explores the relationships of the scale obtained with the variables further approximate the emotional dimensions of individuals. The third part observes the variables that contribute to changes in the scale: These variables show the structural feature of the ontological security.

Key words: emotions; scale; trust; confidence; late modernity; security.

\section{Referencia normalizada}

Callejo J. (2016): "Buscando determinantes de la seguridad ontológica en la España de la crisis", Política y Sociedad, 53 (2), pp.381-412.
\end{abstract}

Sumario: 1. Presentación. 2. El regreso de lo reprimido: la vuelta sociológica a las emociones y la 
seguridad ontológica. 3. El concepto de seguridad ontológica y su potencial para la operacionalización. 4. Metodología. 5. Las narraciones sobre la inseguridad general y la seguridad del mundo materno.

6. Probando la escala: actitudes y confianza. 7. Las diferencias. 8. Conclusiones. 9.Bibliografía.

\section{Agradecimientos}

Este trabajo debe mucho a las reflexiones, recomendaciones y directas aportaciones del profesor Ramón Ramos Torre, dentro de la colaboración conjunta desarrollada en el proyecto CSO 2010-20235 (subprograma SOCI), de la que es investigador principal.

\section{Presentación}

En el presente trabajo, se propone una escala para el concepto seguridad ontológica, elaborado por Giddens. Para ello, se parte de los resultados de una investigación centrada en las relaciones entre riesgo, incertidumbre y vulnerabilidad en el contexto de la crisis económica en España ${ }^{1}$. Esos resultados se han producido mediante 9 grupos de discusión y la realización de una encuesta con cuestionario estandarizado aplicado telefónicamente a una muestra nacional de 2.408 individuos mayores de 18 años.

Tras dar cuenta de algunos de los principales usos que la investigación sociológica empírica ha hecho del concepto y de las pistas que sobre vivencias y sentimientos de inseguridad generalizada ofrecen los materiales discursivos de los grupos de discusión, el trabajo se divide en tres grandes apartados. En el primero, se ha construido una escala a partir de los resultados obtenidos en la aplicación de distintas baterías de ítems presentes en el cuestionario utilizado.

Teniendo en cuenta que la seguridad ontológica es un concepto de contenido emocional, la segunda parte se adentra en las relaciones de la escala obtenida con las variables que en mayor medida se aproximan a las dimensiones emocionales de los individuos. La observación se fundamenta en la relación de la escala con los ítems presentes en una adaptación de la denominada escala de Perspectiva Temporal y con las preguntas que recogen tanto el grado de confianza de los individuos en distintas categorías de personas y de profesiones, como la confianza depositada en la gente, en el otro generalizado.

La tercera y última parte tiene un doble objetivo que se corresponde con las dos relaciones posibles que puede tener toda variable en un modelo. Por un lado, se observan las variables que en mayor medida y de forma estadísticamente significativa contribuyen a las variaciones de la escala obtenida en el primer paso. Desde tal punto de vista la pregunta es: ¿qué dimensiones de la sociedad -centrándonos especialmente en variables sociodemográficas- contribuyen en mayor medida a la seguridad/inseguridad ontológica operacionalizada en la escala? Por otro lado, la escala se utiliza como ventana para diferenciar distintas categorías sociales en función de su relación con esa seguridad ontológica que operacionaliza.

\footnotetext{
${ }^{1}$ Referencia: CSO2010-20235 (subprograma SOCI), investigador principal: Ramón Ramos Torre (UCM).
} 


\section{El regreso de lo reprimido:}

la vuelta sociológica a las emociones y la seguridad ontológica

Giddens $(1990,1991)$ propone la seguridad ontológica como dimensión general existencial en el mundo social (Giddens, 1991:3). Aun cuando siga una línea que viene de Schutz, Goffman o Garfinkel, sus orígenes pueden situarse un poco más allá de la propia sociología, alrededor de las concepciones existencialistas del psicoanálisis de autores como Laing (Bondi, 2014). Es indudable que se trata de un concepto que se hace eco de ciertos aspectos problemáticos y recurrentes de la subjetividad moderna (Burgess, 2012), capaz de conformar por sí mismo una línea autónoma de estudios e investigaciones (Delehanty y Steele, 2009; Berenskoetter y Giegerich, 2010; Steele, 2005; Mitzen, 2006).

El concepto ha sido recogido en los más diversos campos de aplicación de la sociología, desde la sociología de los medios de comunicación -especialmente a partir de la obra de Silverstone y su búsqueda de la implicación de los medios de comunicación en la vida cotidiana (Silverstone, 1993 y 2006; Cohen y Metzger, 1998; Ostertag, 2010; Georgiou, 2013; Williams, 2011)- hasta la sociología de las migraciones (Hinton, 2009; Harney, 2012) o la sociología de la familia (Broom y Kirby, 2013). También las investigaciones propiamente de seguridad lo han recogido, destacando aquellas que hacen referencia al papel de la seguridad internacional y la presencia o ausencia de amenazas externas, como un factor más en la constitución de la seguridad ontológica de los sujetos. Son estudios que indagan qué entienden los sujetos por inseguridad y cuáles son sus fuentes (Vornanen y Torronen, 2009; Harries, 2008) o, tan específicos, como los que se centran en las relaciones chino-japonesas (Gustafsson, 2014).

Sin embargo, es en los estudios que abordan su relación con la vivienda donde hay una mayor concentración de investigación que sitúa a la seguridad ontológica como concepto central. En la mayoría de estos estudios, se parte de la asunción de la propiedad de la vivienda como fuente de seguridad ontológica, siendo notable su variedad temática: el significado de la propiedad (Dupuis y Thorns, 1998), categorías sociales en continuo desplazamiento de residencia (Taylor, 2013), el papel de los préstamos hipotecarios (Ross y Squires, 2011), la pérdida de la vivienda tras una catástrofe natural (Hawkins y Maurer, 2011) o formas de vivienda no convencional (Newton, 2008). Por su parte, en los estudios de género se utiliza también el concepto, relacionándolo con la emancipación (Roe, 2014) y con la violencia de género (Rajah, 2010).

En cuanto a su contenido, se ha destacado su fuerte componente emocional. De hecho, Giddens define el concepto como un sentimiento. Así, puede decirse que este concepto se incluye en la revalorización sociológica de conceptos -como confianza, temor, sentimiento de inseguridad, etc.- entroncados con las emociones, que recupera propuestas ya clásicas aparecidas en la proto-sociología de Tarde, (1989 [1901]:90) o Le Bon (2000 [1895]:18), o en la sociología de Elias (1987)².

${ }^{2}$ La sociología retoma la relevancia de las emociones al final siglo XX, impulsada por Hochschild, Kemper y Scheff. Esta línea hace hincapié en la cultura como elemento fundamental para comprender la formación y la expresión de las emociones (Gordon 1981; 1989), hasta el explícito objetivo de situar las emociones en la sociología (Lemmings y Brooks, 
Sobre la seguridad ontológica como emoción específica, cabe destacar los trabajos de Pretorius (2008), quien, a partir de una investigación desde la perspectiva cualitativa, observa los sentimientos de inseguridad ontológica entre víctimas de robos y asaltos con violencia. Es en la relación entre seguridad ontológica y miedo al delito donde se centra especialmente la atención. A este respecto, hay que resaltar, en el campo de la investigación criminológica, las aportaciones de Kury (1996), que relacionan los sentimientos de inseguridad en general y el miedo al delito en particular, o la de Walklate (2005), para quien el sentimiento de seguridad está mediado por la integración en la comunidad local.

La mayor parte de las observaciones empíricas sobre la seguridad ontológica referenciadas quedan integradas en campos específicos y circunscritos, renunciándose a un abordaje integral del concepto que articule diversos campos, y analice el peso relativo de cada uno en su producción o reproducción. Tal carencia es parcialmente fruto del hecho de que la mayor parte de las aproximaciones se hayan realizado desde la perspectiva cualitativa, quedando apartado el esfuerzo de operacionalizar el concepto y establecer una graduación del mismo. Ese es el objeto central de este trabajo, que ha de empezar, como no podría ser de otra manera, analizando las diversas características del concepto, especialmente siguiendo la obra de Giddens.

\section{El concepto de seguridad ontológica y su potencial para la operacionalización}

No es usual la utilización del término ontología en los trabajos sociológicos. Es más, si apareciera en el título de un artículo, sin mayores referencias, es fácil que lo situáramos en el campo de la filosofía. Por ello conviene aclarar cuál es su papel en la obra que lo elabora inicialmente, la de Giddens. ${ }^{3}$

2014). Asimismo, se apunta la presencia de emociones en algunos de sus campos concretos de estudio. Hochschild observa las dinámicas de la emoción en campos como el trabajo, el consumo o la familia (Hochschild, 1979, 1983 y 1989). Paulatinamente se extiende el interés por las emociones en otros campos, como las prácticas económicas (Smelser, 1998; Berezin, 2009), los movimientos sociales (Emirbayer y Goldberg, 2005; Jasper, 2011), la opinión pública (Brader et al., 2011), cobrando especial relevancia en los estudios sobre las relaciones entre los géneros, desde las aportaciones del propio Hochschild hasta las más recientes (Wharton, 2009, por todos). En la reciente institucionalización sociológica de la observación de las emociones, cabe establecer los textos editados por Kemper (1990), Turner (1999) y por Turner y Stets (2005), que puede considerarse la obra que muestra de una forma más extensiva el campo de la sociología de las emociones, así como el hecho de constituir una voz (Bericat, 2012) en la sociopedia de la ISA (Asociación Internacional de Sociología).

${ }^{3}$ Antes de Giddens (1984, 1990, 1991 y 1992) y de alguno de los autores en que él mismo se apoya, como Schutz (1974a:97), hay que remontarse al trabajo que Lukacs (2007) sobre la obra de Marx, como intento de basar la historia y, de forma indirecta, la sociología en una ontología. Al optar por el sentimiento de seguridad ontológica como algo que atraviesa los diferentes campos del mundo de la vida de los sujetos, pero que, a la vez, diferencia a las distintas categorías sociales, subrayando así su carácter social, hay que resaltar la influencia de 
La que Giddens propone es una ontología de un ser desvalido, que lo primero que busca es protección, seguridad, a través de la sociedad o, con mayor precisión, los representantes próximos de la sociedad, como es la madre. Con la seguridad, con la obtención de mayor o menor grado de seguridad y en continua tensión, el sujeto se construye y se transforma. No obstante, cada sociedad dota de distinta estabilidad a sus individuos, que, por su parte, proyectan sobre ella distintas expectativas. Es aquí donde entra tanto la propia teoría de la modernización de Giddens, como un análisis diferencial de la seguridad ontológica en la estructura de las sociedades. Su teoría de la modernidad explica la relativa debilidad de la seguridad ontológica, que afecta a todos por igual: nadie escapa a una cierta inseguridad (Giddens, 1991:125).

Como tal ontología, la seguridad ontológica es del orden del ser o, todo lo más, del estar en el mundo - tal vez pensando o sintiendo ese mundo- pero se aleja del hacer, de la acción en cuanto agencia. Es una cuestión importante a la hora de la operacionalización del concepto, pues pone en un primer plano las preguntas sobre el ser, sobre cómo se autoperciben y se autodefinen (Kinnvall, 2004) los individuos, sobre sus sentimientos o sobre actitudes hacia el mundo y preocupaciones, dejando en un segundo plano las relativas a su comportamiento y agencia.

El término ontológico tiene en Giddens dos proyecciones. Una como consideración del sentimiento de seguridad como algo básico, que constituye al sujeto y, al permitirle ser-estar, le abre a la acción. Se trata de una especie de matriz que permite que los individuos se enfrenten al mundo (Giddens, 1992). Por otro lado, y en cuanto tal matriz, es algo que atraviesa el total de la relación de los sujetos con el mundo, de manera que sería difícil entenderlo como un sentimiento ubicado en circunscritos aspectos o campos del mundo de la vida, pero ausente de otros. Por lo tanto, la seguridad ontológica es más algo de orden general, es decir, un recurso o forma de enfrentarse a ese mundo en su conjunto, que una forma de responder a determinadas fuentes específicas de seguridad/ inseguridad ${ }^{4}$.

Tal como la concibe Giddens, la seguridad ontológica está relacionada con un mundo próximo y despreocupado, donde todo se espera que funcione y la trayectoria vital del sujeto no está en riesgo o puesta en juego. ${ }^{5}$ Por contra, en el mundo moderno, y tal

Goffman en el concepto de Giddens. Así, toma la figura del jugador, capaz de ver oportunidades donde otros solo ven rutinas (Giddens, 1991:132).

${ }^{4}$ Hay que hacer notar que tal articulación de respuesta emocional general frente a las distintas fuentes de riesgo es contraria a las propuestas de otros autores, como Robert Castel, que al abordar la creciente incertidumbre de nuestro tiempo aboga más por situarlas en fuentes distintas y separadas -categorizadas en tres grandes grupos o factores o tipos de riesgo: riesgos sociales, de las poblaciones de riesgo, tecnológicos- aunque sean todas producto de una dinámica de descolectivización (décollectivisation) o individualización. Apuesta por distinguirlos, en lugar de sumarlos (Castel, 2009: 37).

${ }^{5}$ Esta relación con la falta de desasosiego y preocupación será central a la hora de la operacionalización que se propone del concepto, de manera que permite asimilar inseguridad con preocupaciones y seguridad con cierta falta de preocupaciones. 
como muchos otros sociólogos habían ido mostrando con anterioridad ${ }^{6}$, nos topamos de continuo con un ser humano que se siente inseguro, desconfía y está atravesado por incertidumbres que empantanan su mundo cotidiano de vida. Un retrato así lo podemos remontar a la obra de Marx, especialmente la centrada en la problemática de la alienación (Luckács, 2007).

La relación entre confianza y sentimiento de seguridad es central en la propuesta de Giddens. Se convierte en materia de reflexión y generación de preguntas que sin embargo no consiguen sólidas respuestas y a veces se limitan a aventurar simples apuntes con ideas borrosas o poco definidas. Por ejemplo, Giddens no aclara suficientemente hasta qué punto un sentimiento de seguridad genera desconfianza hacia los demás. No hay que olvidar que, precisamente, cierta seguridad puede llevar a la desconfianza hacia los demás o las instituciones, tal como argumenta de manera convincente Luhmann (1996). Pues es cierto que una confianza básica es necesaria para obtener un inicial sentido de seguridad ontológica - como plantea Giddens, apoyándose inicialmente en la obra de Erikson (Giddens, 1984: 53 y ss)-, pero no está tan claro lo contrario, es decir, que cierto sentido de seguridad sea un marco que produzca confianza concreta, en personas, categorías sociales o instituciones. Es más, podemos representarnos sin dificultad la figura de un individuo (aparentemente) seguro de sí mismo que, a la vez, observa con cierta desconfianza a los demás y los sistemas expertos que le rodean.

Por otro lado, la seguridad ontológica conlleva cierto grado de auto-identidad, de una concepción de sí mismo segura ante los demás y, por lo tanto, de reflexión sobre lo que se es y, conectado -como hace el propio Giddens- con los sentimientos de culpa y especialmente vergüenza, sobre lo que se pretende ser ante los demás, de lo que se es y obtiene eco en el entorno social. La autoidentidad no es solo narrativa, como apunta el propio Giddens (1991), sino conciencia de que se es (o no se es) o adscripción a narrativas existentes. No es solo una narrativa autobiográfica, intransferible, sino conciencia de

\footnotetext{
${ }^{6}$ Esto deriva de la propia concepción de la modernidad que tiene el autor. Nombraremos aquí sólo alguno de sus rasgos: a) autonomía del yo, sometido a una continua reelaboración narrativa, lo que conlleva reelaboración de las trayectorias sociales, lo que, a su vez, hace frecuentes los escenarios y circunstancias en que los sujetos han de tomar decisiones sobre tales trayectorias (fateful moments), y un continuo esfuerzo cognitivo y emotivo del yo; b) reflexividad institucional, como continua puesta entre paréntesis de la vida social y sus rutinas, para transformarlas, no debiéndose olvidar el papel que tiene la rutina en la configuración de la propia seguridad ontológica; c) proyección hacia el futuro, lo que Giddens denomina colonización del futuro. La articulación de, al menos, estos tres rasgos, abren un mundo moderno de incertidumbre y necesidad de tomar decisiones trascendentales, con múltiples alternativas, y que afectan directamente a ese sentimiento de seguridad: "En un orden postradicional, la narrativa del yo se ha visto de hecho reelaborada continuamente y las prácticas de la vida se alimentan con ella, si se quiere que el individuo combine la autonomía personal con un sentido de la seguridad ontológica” (Giddens, 1992: 75).
} 
ser de una determinada manera, de aceptación de sí mismo y de presentación de sí mismo a los demás?

\section{Metodología}

En este trabajo se pretende, a la vez, ir más allá y permanecer en la estela de Giddens, apostando por la posibilidad de que la seguridad ontológica, pese a su complejidad conceptual, pueda observarse empíricamente. Aquí se plantea hacerlo a partir de los resultados de una investigación que ha utilizado tanto la perspectiva cualitativa como la perspectiva cuantitativa de la investigación social. La primera se plasmó en la realización de unos grupos de discusión; la segunda, en el diseño de una encuesta con administración de un cuestionario estandarizado.

Los grupos fueron diseñados en función de distintos contextos de seguridad/riesgo pues, aunque la hipótesis de partida era que los sentimientos de seguridad de sujetos y las categorías sociales se proyectan de manera global y relativamente coherente en todos los ámbitos de la vida, no podía partirse de posiciones esencialmente descontextualizadas con respecto al riesgo (Douglas y Waldasky (1982: 73). El diseño de los grupos fue el siguiente:

- GD1: Funcionarios capital provincia sin riesgos tecnológicos. Hombres. 50/60 años. Valladolid.

- GD2: Técnicas y profesionales, en empresas medias. Mujeres 35/45 años. Toledo.

- GD3: Estudiantes universitarios, clase media-alta. Hombres y Mujeres. Valladolid.

- GD4: Amas de casa, hábitat macrourbano, clase media, con hijos menores. 35/45 años. Madrid.

- GD5: Trabajadoras (dependientas, peluqueras, etc.), hábitat macrourbano, clases populares, con hijos menores. 30/40 años. Madrid.

- GD6: Autónomos (sector oficios: fontanero, taxista, dueño pequeño bar, etc.) entorno urbano. Hombres. 40/50 años. Madrid.

- GD7: Empleados con contrato fijo en empresas grandes o medianas, en proximidad de riesgos tecnoindustriales. Hombres y mujeres. 30/40 años. Tarragona.

- GD8: Trabajadores sector industrial-químico. Hombres. 45/55 años. Madrid.

- GD9: Jóvenes empleados, con contrato temporal. Hombres y mujeres. 20/30 años. Madrid.

${ }^{7}$ Esto ha de tener su proyección en el proceso de obtención de la escala-objetivo central de este trabajo- poniendo el foco especialmente en el tratamiento de las respuestas de aquellas preguntas que requieren una autodefinición del sujeto. Las dos caras de la seguridad ontológica de Giddens están así presentes. Por un lado, como sentimiento, constituido en objeto central de este trabajo. Por otro lado y en la medida que se proyecta en autoidentidad -en presentación a los demás como seguro- como elemento a tener en cuenta en la observación empírica ya que, especialmente entre ciertas categorías sociales, hay una tendencia a manifestarse más seguro de lo que se siente. 
Los grupos de discusión fueron realizados entre los meses finales de 2012 y los iniciales de 2013, con un guión que dejaba una parte muy abierta al principio, tras plantear como estímulo de la reunión cómo veían su futuro en los próximos años. La perspectiva cualitativa tiene una mayor capacidad para introducirse en las vivencias y expresiones de la vida cotidiana, y producir narraciones que plasman expectativas y preocupaciones inmediatas, esperanzas e incertidumbres más próximos, cuando los individuos son cuestionados de una manera abierta sobre el futuro.

Por su parte, la encuesta con cuestionario estandarizado se nutrió en parte del material discursivo recogido, siendo su diseño el siguiente.

- Encuesta con cuestionario estandarizado. Aplicación telefónica.

- Población española mayor 18 años

- Selección aleatoria de las unidades muestrales, con establecimiento de cuotas.

- Cuotas: Edad, sexo (50/50), comunidad autónoma (proporcional), tamaño hábitat (proporcional) y ocupación (44\%)

- Tamaño muestral diseñado 2400 individuos. Tamaño final total: 2408.

- Trabajo de campo: mayo 2014

- El cuestionario contó con un total de 41 preguntas, algunas de ellas tomando la forma de batería de ítems, con escala.

- La duración media de las entrevistas finalizadas fue de 25 minutos y 37 segundos.

El cuestionario resultante recoge una amplia gama de preguntas que se introducen en el grado de vivencia de ciertos fenómenos generales que pueden considerarse amenazantes en nuestras sociedades, los sentimientos ante los mismos y, especialmente a partir de una reformulación de la escala de Perspectiva Temporal de Zimbardo (Zimbardo y Boyd, 1999), la autoidentidad del sujeto en contextos de decisión, de esas decisiones importantes en las que parece jugarse buena parte del destino de la vida (fateful), y que, como el propio Giddens señala (1991: 14), son características de la Modernidad y que, por lo tanto, los entrevistados reciben de forma prácticamente "natural". No obstante, no puede definirse como un cuestionario sobre riesgo al uso, pues queda lejos de los cuestionarios centrados en específicas fuentes de riesgo, en los que se suele asumir que los encuestados tienen una suficiente información sobre los mismos y la procesan según niveles distintos de racionalidad (Douglas y Waldasky, 1982: 69). La encuesta con cuestionario estandarizado, como el referido, es un buen instrumento de partida para abordar la seguridad ontológica en la medida que se asume como un factor latente en la vida de los individuos que, sin embargo, puede tener expresión en declaraciones de distintas actitudes ante la vida, el futuro, de preocupaciones sobre ciertas dimensiones de la vida social o sobre la propia identidad. El cuestionario utilizado aborda todos estos aspectos.

A pesar de que el estudio pregunta por las preocupaciones e incluso por los miedos de la gente -aun cuando de una manera bastante indirecta, tanto en la perspectiva cualitativa como en la cuantitativa- se aleja de los estudios de victimización al uso, destinados a fundamentar acciones de control del crimen. El foco aquí es la observación de los temores, preocupaciones o, si se quiere, preocupaciones negativas que tienen los 
sujetos; pero no para detectar (posibles) víctimas, sino para establecer las relaciones entre los distintos temores.

Las operaciones que se han llevado a cabo con el material resultante de la aplicación del cuestionario han ido dirigidas a la construcción de una escala de seguridad ontológica (ESO). La estrategia desarrollada ha sido la siguiente.

Se partió de un análisis factorial de componentes principales ${ }^{8}$ en el que, inicialmente se introdujeron los elementos escalares pertenecientes a la pregunta 2 (grado en que preocupan una serie de temas, tras inversión de la escala ${ }^{9}$ ), pregunta 6 (grado de confianza en categorías de personas ${ }^{10}$ ), pregunta 7 (grado de confianza en profesiones ${ }^{11}$ ), pregunta 8 (grado de acuerdo con determinadas frases, en adaptación de la escala de Zimbardo, haciendo que todos los ítems fuesen en el mismo sentido ${ }^{12}$ ), pregunta 11 (grado de preocupación ante la posibilidad de verse afectado por distintos tipos de catástrofe ${ }^{13}$ ) y

${ }^{8}$ El análisis factorial es la técnica multivariante pertinente para aproximarse a la estructura profunda y las interrelaciones de las variables.

${ }^{9}$ La pregunta general fue: "le voy a ir nombrando una serie de temas, dígame el grado en el que le preocupan siendo 0 igual a 'no le preocupan nada en absoluto' y 10 'le preocupan muchísimo', siendo los ítems concretos: el calentamiento global y el cambio climático, la pobreza, el futuro de las pensiones, la contaminación del aire o del agua, el trabajo, la propagación de enfermedades infecciosas, la adicción a las drogas, el aumento de la población mundial, los accidentes de tráfico, los residuos tóxicos o atómicos y la delincuencia.

${ }^{10}$ La pregunta general fue: "Teniendo en cuenta una escala que va desde 0 , que significa ninguna confianza, hasta 10 , total confianza, dígame el grado de confianza que tiene usted en...", siendo los ítems concretos: personas que acaba de conocer, sus vecinos, sus amigos, sus familiares más directos como padres, hermanos o abuelos, otros familiares más lejanos como tíos, primos, sobrinos, suegros, yernos, nueras o cuñados. Hay que hacer notar que el cuestionario incluía dos ítems más: compañeros de trabajo o estudio e hijos; pero no formaron parte de la construcción de la escala porque implicaban exclusión de buena parte de la muestra original, como los que no trabajan ni estudian o los que no tienen hijos.

${ }^{11}$ La pregunta general fue: "Igualmente, desde un mínimo de 0 a un máximo de 10 , el grado de confianza que tiene usted en los siguientes colectivos...", siendo los ítems concretos: los científicos, los sacerdotes, los médicos, los periodistas, los psicólogos, los jueces, los maestros y profesores, los ecologistas, los bomberos, los empresarios y los policías.

${ }^{12}$ Fueron incluidas en la construcción de la muestra las frases: "El futuro es tan incierto que me parece mejor vivir al día", "Poco importa lo que haga porque lo que tenga que ser, será", "Las cosas cambian tanto que el futuro no se puede planificar", "Por mucho que uno se informe y calcule, al final ocurrirá lo que tenga que ocurrir", "No tiene sentido preocuparse por el futuro porque no se puede hacer nada por cambiarlo", "Me gusta que las cosas estén seguras y no haya sorpresas", "La seguridad al cien por cien no es posible, pero hay que intentar alcanzarla", "Cuando he sufrido una desgracia, he tardado mucho en recuperarme", "En la vida, que las cosas salgan bien o mal depende de la suerte".

${ }^{13}$ La pregunta general fue: “¿Cuál es su grado de preocupación, entre 0 y 10 , siendo 0 igual a nada preocupado y 10, totalmente preocupado, ante la posibilidad de verse afectado por una catástrofe de tipo...?", siendo los ítems: natural (terremoto, inundación o incendio, etc.), tecnológico (accidente industrial o química, accidente nuclear, transporte, etc.), de 
pregunta 19 (percepción del grado de exposición personal a distintas fuentes de riesgo, invirtiendo la escala desde su exposición en el cuestionario $\left.{ }^{14}\right)^{15}$.

Tabla 1: Escala de Seguridad Ontológica resultante con la correlación de cada uno de los ítems con que se ha construido

\begin{tabular}{|l|l|c|}
\hline \multirow{4}{*}{ Pregunta } & Ítems & $\begin{array}{c}\text { Correlación entre } \\
\text { ítems y factor/ } \\
\text { componente }\end{array}$ \\
\hline \multirow{5}{*}{$\begin{array}{l}\text { Grado de preocupación } \\
\text { por... }\end{array}$} & Calentamiento global y cambio climático &, 543 \\
\cline { 2 - 3 } & Pobreza &, 513 \\
\cline { 2 - 3 } & Mantenimiento de pensiones &, 482 \\
\cline { 2 - 3 } & Contaminación &, 570 \\
\cline { 2 - 3 } & Trabajo &, 422 \\
\cline { 2 - 3 } & Epidemia &, 661 \\
\cline { 2 - 3 } & Droga &, 611 \\
\cline { 2 - 3 } & Aumento de la población &, 408 \\
\cline { 2 - 3 } & Tráfico &, 619 \\
\cline { 2 - 3 } & Residuos tóxicos o atómicos &, 677 \\
\cline { 2 - 3 } & Crimen, delincuencia &, 638 \\
\hline
\end{tabular}

violencia (atentado terrorista o conflicto bélico), de convivencia (accidente por asistir a actos o espectáculos masivos).

${ }^{14}$ La pregunta general fue: "De los peligros a los que la gente puede estar expuesta y por la vida que usted lleva, dígame en qué grado -entre 0 y 10 - cree usted que está expuesto a los siguientes peligros", siendo los ítems: sufrir un accidente de tráfico, ser víctima de un robo o atraco, sufrir una enfermedad crónica, quedarse sin el dinero necesario para cubrir las necesidades básicas o de su familia, resultar intoxicado por comer alimentos contaminados, sufrir un accidente muy grave en el desempeño de su trabajo y quedarse sin vivienda.

${ }^{15}$ No se ha tenido en cuenta la no respuesta en aquellas preguntas cuyas respuestas suponen una expresión de autoidentidad, de conocerse y mostrarse a los demás, en la medida que la propia no respuesta podía interpretarse como una falta de autoidentidad. Tal decisión viene motivada porque el porcentaje de no respuestas ha sido muy pequeño, no llegando al $1 \%$. Por otro lado, tan baja proporción de falta de respuestas puede entenderse como indicador de: a) la comprensión de las preguntas; b) el hábito incorporado por parte de los individuos de la autodefinición, pues buena parte de las mismas se encontraban en tales propuestas de autodefinición. 


\begin{tabular}{|c|c|c|}
\hline Pregunta & Ítems & $\begin{array}{c}\text { Correlación entre } \\
\text { ítems y factor/ } \\
\text { componente }\end{array}$ \\
\hline \multirow{4}{*}{$\begin{array}{l}\text { Preocupación ante } \\
\text { posibilidad de verse } \\
\text { afectado por catástrofe... }\end{array}$} & Natural & ,682 \\
\hline & Tecnológica & 689 \\
\hline & Violencia & 685 \\
\hline & Convivencia & ,569 \\
\hline \multirow{6}{*}{$\begin{array}{l}\text { Percepción grado de } \\
\text { exposición a... }\end{array}$} & Accidente de tráfico & ,422 \\
\hline & Robo o atraco & ,475 \\
\hline & Enfermedad crónica & ,416 \\
\hline & Quedarse sin dinero & ,465 \\
\hline & Intoxicación alimentaria & ,417 \\
\hline & Pérdida de vivienda & ,393 \\
\hline \multirow{9}{*}{$\begin{array}{l}\text { Frases sobre formas de } \\
\text { entender la vida... }\end{array}$} & $\begin{array}{l}\text { El futuro me parece tan incierto, que es mejor vivir al } \\
\text { día }\end{array}$ &, 218 \\
\hline & $\begin{array}{l}\text { Poco importa lo que haga porque lo que tenga que ser, } \\
\text { será }\end{array}$ & ,344 \\
\hline & $\begin{array}{l}\text { Las cosas cambian tanto que el futuro no se puede } \\
\text { planificar }\end{array}$ & ,256 \\
\hline & $\begin{array}{l}\text { Por mucho que uno se informe y calcule, al final } \\
\text { ocurrirá lo que tenga que ocurrir }\end{array}$ & ,335 \\
\hline & $\begin{array}{l}\text { No tiene sentido preocuparse por el futuro porque no se } \\
\text { puede hacer nada por cambiarlo }\end{array}$ & ,246 \\
\hline & $\begin{array}{l}\text { Me gusta que las cosas estén seguras y no haya } \\
\text { sorpresas }\end{array}$ & ,209 \\
\hline & $\begin{array}{l}\text { La seguridad al cien por cien no es posible, pero hay } \\
\text { que intentar alcanzarla }\end{array}$ & ,201 \\
\hline & $\begin{array}{l}\text { Cuando he sufrido una desgracia, he tardado mucho en } \\
\text { recuperarme }\end{array}$ &, 270 \\
\hline & $\begin{array}{l}\text { En la vida, que las cosas salgan bien o mal depende de } \\
\text { la suerte }\end{array}$ &, 253 \\
\hline
\end{tabular}

Fuente: Elaboración propia a partir de la encuesta realizada.

El análisis se fijó en el primer componente extraído. A partir de ahí, se pasó a eliminar los ítems que en la matriz del componente aparecían con valores muy bajos, ya fueran negativos o positivos. No obstante, se han mantenido algunos ítems, a pesar de su baja correlación con el factor obtenido, por su significatividad, por el sentido que dan a la escala y, teniendo en cuenta que el tamaño muestral utilizado y el número de variables 
analizadas, porque cargas factoriales o correlaciones entre factor y variables por encima de 0,2 pueden considerarse significativas.

Hay que señalar que hubo que eliminar todos aquellos ítems inicialmente introducidos relacionados con la confianza, lo que apunta primeramente a que la relación entre nuestra escala de seguridad ontológica (ESO) y la confianza dista de ser lineal, y, en segundo lugar y teniendo en cuenta que la correlación entre el componente principal obtenido y los ítems relativos a la confianza - con una escala desde 0 o ninguna confianza a 10 o máxima confianza- era negativa, se planteaba la necesidad de excluirlos de la escala para observar posteriormente cuáles eran las relaciones entre la propia ESO y las escalas de los ítems de confianza. Por lo tanto, se invitaba a una reflexión sobre confianza y seguridad ontológica, que, por otro lado, está en el centro de la propuesta de Giddens.

Se observa (Tabla 1) que en la escala resultante tienen mayor correlación los ítems vinculados a la percepción de ser afectado por algún tipo de catástrofe o aspectos de la vida cotidiana. El porcentaje de varianza explicada por este primer componente principal es $22,893 \%$. El estadístico de fiabilidad utilizado, el alfa de Cronbach que valora la consistencia de la escala entera, para estos 30 ítems o elementos, es alto: 0,874 ${ }^{16}$, superando ampliamente el límite inferior (Hair et al., 1999:105 y 116). Ahora bien, más allá de la coherencia en la construcción del instrumento, parece que lo relevante es el grado de validez y, por lo tanto, de ajuste al propio concepto de seguridad ontológica, tal como es propuesto por Giddens. Es lo que se desarrolla en la segunda parte de este trabajo, tras detenerse en la observación de la relevancia que tiene el tema de la seguridad en el discurso espontáneo de los sujetos. Sin embargo, antes hay que apuntar la existencia, en el propio cuestionario, de puntos de referencia con el objetivo de poder calibrar, aun cuando sea parcialmente, tal validez. Así, aun cuando, como se ha dicho, preguntar más o menos directamente por el sentimiento de seguridad que tienen los individuos en el contexto inmediato de la aplicación de un cuestionario puede estar fuertemente condicionado por la presión de una tradición cultural que prima el mostrarse seguro, el uso de una pregunta de tal cariz puede tener la

\footnotetext{
${ }^{16}$ Por otro lado, se obtiene una correlación positiva $(0,576)$ y estadísticamente significativa (en el nivel 0,01, 2 colas) con una escala aditiva formada por los siguientes ítems: "El mundo será mejor para la próxima generación" ( $\mathrm{p} .1=1$, mejor), "Baja preocupación distintos temas" (p.2<8),"Sentimiento seguridad aspectos vida personal" (p.3=1, seguro), "Confianza en el otro generalizado (p.5=1, la mayoría de la gente es de fiar)", "Confianza entorno relacional" (p.6>6), "Confianza profesiones" (p.7>6), "Determinación consecución metas (p.8.2=1,2, totalmente/ bastante)", "Correr riesgos por emoción" (p.8.4=1,2), "Hay que intentar conseguir la seguridad total (p.8.12=1,2)", "Aprovechar las oportunidades, aunque suponga riesgos" (p.8.13=1,2), "Tardanza en recuperación tras desgracia" (p.8.14=3,4, poco/nada), "Consecución de logros mediante el esfuerzo" (p.8.16=1,2), "Visión del futuro sin preocupaciones o miedos" (p.9=1,2, muy/bastante segura), "Preocupación por catástrofes" $(p .11<4)$, "Beneficios desarrollo

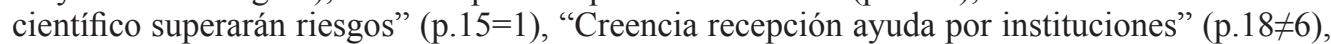
"Percepción de exposición a peligros" (p.19<4), "Desgracias en entorno" (p.20=2) y "Ayuda en situación necesidad" (p.21>0).
} 
función de confirmación parcial -siempre que vayan en el mismo sentido las respuestas a esa pregunta y la escala producida- de estar en un camino acertado, por lo que se ha incluido en el análisis aquí realizado.

\section{Las narraciones sobre la inseguridad general y la seguridad del mundo materno}

El estudio empírico se llevó a cabo en plena crisis económica en España. Puede asumirse que, al menos, los grados de incertidumbre eran altos. Por otro lado, el diseño de los grupos de discusión realizados se llevó a cabo asumiendo que sus participantes respectivos ocupaban posiciones y distancias distintas con respecto a varias fuentes de riesgo, desde la aparente preservación ante riesgos del grupo de mujeres en una pequeña ciudad como Toledo o del grupo de empleados del sector público de Valladolid, hasta los grupos de jóvenes o de trabajadores autónomos, con asunción de fuertes riesgos sobre su cabeza. Pero, más allá de las diferencias y atendiendo a la idea de que la inseguridad -en la sociedad moderna- es una especie de ethos cultural que atraviesa las distintas posiciones: ¿es la seguridad un problema para los sujetos?

En tal contexto, los discursos recogidos a partir de una dinámica inicialmente muy abierta, se hacen eco de lo que podría considerarse cierto grado de ansiedad:

- “...es la [crisis] que más nos está asustando" (GD.1)

- "Porque todos tenemos el miedo de... estoy en paro... me echarán, si tienes trabajo" (GD.5)

- "Pero se te ha metido el miedo en el cuerpo" (GD.6)

- "Porque de repente se nos han caído todos los esquemas" (GD.8)

- "¿Y quién ha generado esto? ¿para qué? Para meterte el miedo en el cuerpo" (GD.8)

Un sentimiento general -obsérvese la diversidad de posiciones sociales recogidas- de inseguridad, dominado por el miedo y el reconocimiento de que los esquemas, término que hay que considerar próximo al de estabilidad espacio-temporal de Giddens, se han caído. Se trata de la experiencia de un sentimiento de inseguridad que aumenta cuando en los discursos aparecen los hijos. Entonces, se convierte en miedo por los hijos y las dificultades para establecer vías para la denominada colonización del futuro. Una situación en la que el sentimiento de inseguridad va en paralelo con algo que el propio Giddens describe (especialmente en Giddens, 1991), la sensación de falta de poder, de impotencia: "Tenemos inseguridad y sensación de impotencia ¿no?" (GD.4).

Por lo tanto, la inseguridad aparece con una referencia general. En lo concreto, la única fuente especificada es el ámbito laboral, la inseguridad en el trabajo. Pero siendo ésta importante, no podría decirse que es la fuente que monopoliza la generación de inseguridad, de tal manera que en los otros ámbitos de la vida se viviera con seguridad. Y esto a pesar de que otras potenciales fuentes no aparecen espontáneamente en el discurso como tales fuentes de inseguridad y cuando aparecen, de la mano de la moderación de las reuniones, son representadas como fuentes de inseguridad de baja intensidad -problemas urbanos, de delincuencia, medioambientales, de relaciones de 
pareja o convivencia- a pesar de esa sensación de inseguridad general expresada en términos de un desamparo institucional: "No te amparan las instituciones" (GD.2). Lo que antes aparecían como sistemas que "aseguraban cuestiones" (GD.2), la crisis económica los ha convertido en vulnerables en sí mismos.

Se tiene una sensación de inseguridad generalizada que bloquea la posibilidad de dar pasos hacia el futuro: "Prefiero seguir aqui por si acaso me renuevan" (GD.9). Las expresiones de inseguridad se proyectan espontáneamente hacia el futuro con notable fuerza semántica y, como se decía antes, personalizando en los hijos:

- "En el fondo estamos hablando todo el rato y si te das cuenta, nadie quiere hablar del futuro. Estamos todos aquí atrincherados diciendo: mientras mañana salga el sol" (GD.6)

- "Antes se hacían las cosas así, mañana no sabemos lo que va a pasar" (GD.1)

- "yo por ejemplo el futuro de los críos, sí te da miedo, porque piensas en estas cosas de decir, pues, por ejemplo, yo creo que se está subiendo el tema de sanidad, el tema educación [...] Cuando lleguen nuestros hijos a una edad ¡qué será de ellos!'(GD.5)

- "No veo mi futuro ¡Como para ver el de las niñas!” (GD.6)

- "Le va a afectar a tu hijo, le va a afectar al mío, le va a afectar a todo" (GD.8)

En un mundo representado como inestable, recobra fuerza el entorno inmediato, regresivo, de la casa de los padres -y, especialmente de la madre- como base de la seguridad ontológica. El miedo, en cuanto se abre un abismo con su carencia, está en la pérdida de padres y madres:

- "A mí me da mucho miedo tal vez dejar a mi familia [...] porque siempre puedes volver" (GD.3)

- "...muchas veces discutes con tu pareja y dices: que me largo... siempre sabes que tienes la casa de tus padres...[...] Si no puedes pagar el alquiler, te vuelves con ellos" (GD.8)

- "Yo tengo más miedo que le pase algo a mis padres, que a no tener yo familia [...] siempre sabes que tienes ahí la casa de tus padres [...] te genera como una seguridad saber que están ahí [...] Si tus padres no están ahí, es cuando te vas a ver solo $[\ldots]$ te genera como una seguridad el saber que están ahí $[\ldots]$ la red de todo el mundo son los padres" (GD.9)

Los padres y su casa (o los padres-casas) aparecen como seguridad material -"si no puedes pagar...."- pero, sobre todo, emocional. Expresiones que nos llevan, por la vía regresiva, a ese entorno doméstico material en el que el propio Giddens ubica originalmente la seguridad ontológica, como si fuese la matriz y "la casa" de la seguridad ontológica.

Por lo tanto, está presente una sensación generalizada de inseguridad e inestabilidad - ciertamente compensada parcialmente por la referencia a la estabilidad de los padres, el que estén ahí. La matriz polarizada entre cierta inseguridad y cierta seguridad está presente en los discursos de los grupos. La seguridad aparece espontáneamente como 
problema en los discursos. Pero ¿quiere esto decir que todas las categorías sociales viven en el mismo grado el problema, sienten igualmente esa seguridad/inseguridad de base? Es aquí donde resulta pertinente la escala elaborada.

\section{Probando la escala: actitudes y confianza}

Hay una relación relativamente positiva entre considerarse una persona segura y la seguridad ontológica, puesto que las puntuaciones en la escala de quienes dicen considerarse una persona muy segura o bastante segura son respectivamente de 0,73 y 0,43 ; mientras que entre los que se consideran personas pocos seguras o nada seguras son $-0,15$ y $-0,45$. No obstante, las respuestas a esta pregunta parecen recoger en buena medida la presión cultural a mostrarse seguros en espacios públicos, fuera del entorno más afectivamente cercano, ya que el $79 \%$ de los consultados dice considerase una persona muy o bastante segura. Interpretación que, más allá de la observación de la validez sociométrica del instrumento creado, nos conduce a su validez práctica en la medida que permite abordar el sentimiento de seguridad soslayando parcialmente la fuerte presión cultural a mostrarse como persona segura.

Para reconocer la validez del instrumento, conviene situarse en las figuras extremas de la propuesta conceptual de la seguridad ontológica y ver hasta qué punto se corresponden con la escala resultante. Así, Giddens sitúa en uno de los polos de la seguridad ontológica la figura del paranoico, quien interpreta en términos de amenaza cualquier modificación en sus rutinas y cuya búsqueda de seguridad apunta a la carencia de la misma; en el otro polo, sitúa el colonizador de futuro, el tomador de riesgos (risk taking), seguro de su capacidad para afrontar los riesgos y aprovechar las oportunidades (Giddens, 1991: 133). Pues bien, en la adaptación de la escala de Perspectiva Temporal adaptada en el cuestionario, hay frases que pueden considerarse concreciones de ambas figuras, como se verá.

Hay que resaltar primeramente la ausencia de problemas por parte de los individuos en las muestras de autodefinición y, por lo tanto, de autoidentidad ${ }^{17}$. En segundo lugar, como medida de precaución metodológica, se ha comprobado el grado de presencia de patrones sospechosos de respuesta, por si era necesaria la eliminación de algunos casos. $^{18}$

${ }^{17}$ Tal como se hace en esta investigación, plantear que las emociones y los sentimientos pueden observarse a partir de las expresiones orales de los sujetos, y, por lo tanto, desde cierta racionalización, lo que significa ir más allá de la oposición racional-emocional, como propone Featherstone (1991: 59), y supone aceptar una racionalización de lo emocional y una "emocionalización”, puesto que conlleva una presentación de sí mismo, de actuaciones que se entienden por los sujetos como racionales. Por supuesto, esta aproximación empírica, como toda aproximación a través de la pregunta a los sujetos, ha de ser prudente en sus conclusiones, pues el nudo formado por emoción y razón nunca está totalmente desatado.

${ }^{18}$ Como en la redacción de las preguntas en el cuestionario se fue alternando el sentido de las preguntas -hacia unas posiciones supuestamente más seguras unas, hacia unas posiciones supuestamente más inseguras otras- no cabía que los sujetos, por coherencia, respondieran a 
Para el caso de las posiciones más fatalistas, se ha mostrado la ausencia de seguridad ontológica, según nuestra escala. Así, en los que están totalmente de acuerdo con la frase "Poco importa lo que haga porque lo que tenga que ser, será", la puntuación en la escala desciende al -.59 (frente al .49 de los que no están nada de acuerdo). Algo semejante a lo que ocurre con otras frases, como: "Por mucho que uno se informe y calcule, al final ocurrirá lo que tenga que ocurrir" (-.44, frente al .37 de los que no están nada de acuerdo), "No tiene sentido preocuparse por el futuro porque no se puede hacer nada por cambiarlo" (-.67, frente a .25), "En la vida, que las cosas salgan bien o mal depende de la suerte"(-.71, frente a .27). De hecho, las diferencias entre las puntuaciones máximas en la escala obtenidas en las distintas posiciones con respecto a estas frases se encuentran en: "Por mucho que uno se informe y calcule, al final ocurrirá lo que tenga que ocurrir", con 1,11 puntos de diferencia entre quienes optan por el totalmente de acuerdo y los que optan por el nada de acuerdo, y "Poco importa lo que haga porque lo que tenga que ser, será" $(1,09)$.

Por lo tanto, la escala parece ser capaz de detectar la figura de los fatalistas, identificándolos con una baja seguridad ontológica.

Sin embargo, las diferencias entre posiciones son muy inferiores -incluso no lineales- en las frases que parecen incorporar la figura de los arriesgados. Así, ocurre en: "En la vida, si uno se esfuerza consigue las cosas que se propone" (diferencia de 0,17 , para lo que ha de tenerse en cuenta que la mitad de la muestra dice estar bastante de acuerdo con la frase), "Aprovecho siempre las oportunidades aunque supongan muchos riesgos" (diferencia es de 0,22 ), o "Cuando quiero conseguir algo, me fijo unas metas y pongo los medios para alcanzarlo" $(0,20)$.

Dada la fuerte relación que Giddens establece entre confianza y seguridad ontológica, en los primeros pasos hacia el análisis de componentes principales que tenía como horizonte la construcción de la escala se incluyeron las preguntas sobre el grado de confianza en categorías de personas y profesiones. Sin embargo, se observó que esos ítems correlacionaban en sentido opuesto a los distintos componentes principales que iban surgiendo. Así, puede observarse en la tabla 3, donde solo la confianza en los jueces correlaciona positivamente, aunque con una cifra reducida. Los coeficientes de correlación negativos mayores son con respecto a los vecinos, entre las categorías personales, y periodistas y ecologistas, entre las categorías del sistema experto. Esto nos estaría diciendo que quien muestra cierto sentimiento de seguridad, a partir de la puntuación obtenida en la ESO, tenderá a mostrarse relativa y diferencialmente menos confiado en relación a los vecinos, periodistas, ecologistas, bomberos, psicólogos, etc. De esta forma, la ESO parece operar como un indicador de autonomía de los individuos, de personas que prefieren arreglárselas por sí solas.

En cualquier caso, los resultados de la tabla 3 parecen ir en un sentido distinto a la relación que Giddens establece entre seguridad orgánica y confianza, concebidas como dos realidades emocionales que marchan en paralelo. No parece ser así en el caso de

todos los elementos de la batería con la misma posición: totalmente de acuerdo, bastante de acuerdo, poco de acuerdo o nada de acuerdo. De hecho, solo 19 casos (0,5\% del total) responden a más de las dieciséis sentencias planteadas con el totalmente de acuerdo. 
confianza en perfiles concretos de personas o profesionales. Sin embargo, si se pone la ESO en relación con una confianza menos definida, con perfiles más bajos, como puede ser la confianza en la gente, los resultados son distintos. Entonces, se observa que la frase "la mayoría de la gente es de fiar", que puede entenderse como operacionalización de una confianza general en el mundo y la sociedad, obtiene una puntuación positiva $(0,27$, en tabla 4$)$, mientras que la frase que concreta una desconfianza también generalizada -"Nunca se es lo suficientemente cuidadoso al tratar con la gente"- obtiene una puntuación media negativa.

Tabla 2: Coeficientes de correlación de ítems de confianza con Escala de Seguridad Ontológica

\begin{tabular}{|l|c|}
\hline Confianza en... & $\begin{array}{c}\text { Coeficiente de } \\
\text { correlación de Pearson }\end{array}$ \\
\hline Personas que acaba de conocer &,- 029 \\
\hline Sus vecinos &,$- 117^{* *}$ \\
\hline Sus amigos &,- 011 \\
\hline Sus familiares más directos como padres, hermanos o abuelos &,$- 074^{* *}$ \\
\hline Otros familiares más lejanos como tíos, primos, sobrinos, suegros &,$- 084^{* *}$ \\
\hline Los científicos &,$- 116^{* *}$ \\
\hline Los sacerdotes &,$- 046^{*}$ \\
\hline Los médicos &,$- 103^{* *}$ \\
\hline Los periodistas &,$- 229^{* *}$ \\
\hline Los psicólogos &,$- 181^{* *}$ \\
\hline Los jueces &, 012 \\
\hline Los maestros y profesores &,$- 155^{* *}$ \\
\hline Los ecologistas &,$- 215^{* *}$ \\
\hline Los bomberos &,$- 182^{* *}$ \\
\hline Los empresarios &,- 018 \\
\hline Los policías &,$- 176^{* *}$ \\
\hline
\end{tabular}

** La correlación es significativa en el nivel 0,01 (dos colas)

Fuente: Elaboración propia a partir de la encuesta realizada. 
Tabla 3: Medias en la Escala Seguridad Ontológica

según opciones con relación a la confianza en general

\begin{tabular}{|l|c|c|c|}
\hline & Media & $\mathrm{N}$ & Desviación estándar \\
\hline La mayoría de la gente es de fiar &, 2713593 & 1015 &, 98069723 \\
\hline $\begin{array}{l}\text { Nunca se es lo suficientemente cuidadoso al } \\
\text { tratar con la gente }\end{array}$ &,- 1980084 & 1391 &, 96726657 \\
\hline
\end{tabular}

Fuente: Elaboración propia a partir de la encuesta realizada.

La seguridad ontológica aparece así directamente relacionada con una especie de confianza básica, general, con una confianza que podría decirse que está ubicada en el sujeto, de la que éste es emisor. Sin embargo, lo está inversamente con una confianza que parece apuntar a una falta de autonomía del sujeto, derivando de, al menos, potenciales necesidades, con una confianza que podría interpretarse como confianza necesitada: de los bomberos, temiendo el incendio; de los psicólogos, asumiendo ya la posibilidad de derrumbe personal, etc.

Mientras la confianza general surge de la fortaleza del sujeto, el segundo tipo de confianza tal vez muestra su debilidad. Utilizando la sutil diferencia en inglés entre confidence y trust, ambas trasladables al español como confianza y en buena parte concebidas como sinónimos en el propio inglés, la primera es la que parece más vinculada al sentimiento de seguridad ontológica en la medida que admite entre sus principales acepciones la autoconfianza (belief in one's own abilities) ${ }^{19}$, en las posibilidades propias. Por su lado, trust se proyecta en todas sus acepciones en la alteridad, incluso utilizando el propio término de confidence (trust: reliance on and confidence in the truth, worth, reliability, etc. of a person or thing) ${ }^{20}$, estando más cercana de la confianza más concreta en personas y categorías profesionales. Es cierto que una frase como: "La mayoría de la gente es de fiar", que es la utilizada en el cuestionario, puede ser tomada también como confianza en la alteridad -en ese otro general que es la gente- pero: a) no se trata de un tipo de personas concretas, como ocurre en los otros tipos de pregunta utilizados; b) no es descabellada su interpretación como: "yo soy una persona que se fía de la gente, porque del primero que me fío es de mí mismo y, por lo tanto, soy una persona de fiar". Es decir, la reflexividad en clave de autoconfianza-y, por lo tanto, confidence- está más cerca en esta frase sobre la confianza en general. Como concluye Barbalet (2009) en su intento por definir el concepto de confianza (trust), ésta puede ser traicionada, lo que parece más lejano tanto del término confianza como confidence, como de la frase utilizada en el cuestionario para abordar un sentimiento general de confianza, pues es difícil pensar que: "la gente puede traicionarte".

\footnotetext{
${ }^{19}$ Collins Dictionaries (2014).

${ }^{20}$ Collins Dictionaries (2014).
} 
Más allá del sistema teórico del propio Giddens, son pocos los enfoques empíricos que abordan la relación entre confianza y seguridad ontológica. Por ello, se destaca el de Sandra Walklate (1998). Para esta autora, la respuesta a la pregunta en quién confiar es relevante para entender la gestión de la seguridad ontológica, refiriéndose a una comunidad urbana en los alrededores de Manchester que confía en no ser atacada por los que considera los suyos y desconfía de los que le son ajenos, especialmente de las instituciones. Es la comunidad la que confía en la comunidad, siendo los miembros de una comunidad los que confían en los otros miembros por el mero hecho de serlo. Se plantea un sentido de pertenencia comunitaria y de confianza en relación con la seguridad ontológica que concibe a ésta de una manera particularmente territorializada y local: seguridad ontológica en "mi territorio", junto a la inseguridad que deben sentir los ajenos a tal territorio y el desconocimiento de la proyección de tal seguridad más allá de las fronteras del barrio. Se trata de una concepción de la seguridad ontológica que parece distante de ese sentimiento más o menos profundo de autoconfianza que se proyecta en los más diversos campos de la vida, tal como lo dibuja Giddens. Por otro lado, los rastros de ese sentimiento comunitario no se han encontrado en la aproximación cualitativa de este estudio. En especial cuando la respuesta dominante a la cuestión de en quién o qué se confía fue: "en nadie".

Con el propósito de ahondar en las relaciones entre la ESO y la confianza, poniendo el foco en la confianza en categorías de personas y profesiones, se ha realizado un análisis de conglomerados (cluster). A partir de las baterías de preguntas sobre el grado de confianza en categorías de personas y profesionales, se ha aplicado un análisis cluster o de conglomerados con una salida de cuatro grupos ${ }^{21}$, que son los que aparecen en la tabla 4:

Tabla 4: Puntuaciones en la escala de seguridad ontológica según grupo (conglomerado)

\begin{tabular}{|l|c|c|}
\hline & Porcentaje sobre total muestra & Media \\
\hline Confiados & 29,2 &,- 2272800 \\
\hline Confianza en expertos & 24,6 &,- 0512884 \\
\hline Desconfiados & 16,1 &, 2207911 \\
\hline Confianza de proximidad & 23,5 &, 2370480 \\
\hline
\end{tabular}

Fuente: Elaboración propia a partir de la encuesta realizada.

El grupo de confiados es el que presenta un grado relativamente alto de confianza tanto en categorías personales como profesionales. Constituye el $29,2 \%$ del total de la muestra

\footnotetext{
${ }^{21}$ Se utiliza k-means como algoritmo de agrupamiento, con $\mathrm{k}=4$.
} 
y se relaciona negativamente con la ESO, ya que la puntuación media de este grupo es de $-0,23$. Parece que los confiados, es decir, los que confian sucesivamente en todas las categorías sociales presentadas, tienden a la inseguridad ontológica, a ser inseguros y estar llenos de preocupaciones y sentimientos de ser convertidos en víctimas.

Una segunda agrupación, la denominada de confianza en expertos, se realiza cuando se alcanzan grados relativamente elevados de confianza en los profesionales, pero que, comparados con los otros grupos, no resultan tan elevados en relación con las personas próximas, como en el caso del grupo anterior. Hay que anotar que la media del grado de confianza con respecto a las distintas categorías de profesionales es superior a la que presentan los conglomerados de desconfiados y confiados en los próximos, excepto, en la comparación con estos últimos con respecto a sacerdotes, empresarios y policías. Parece así que los confiados en los próximos incluyen entre tales próximos a sacerdotes, empresarios y policías. El conglomerado de quienes confían preferentemente en expertos supone casi la cuarta parte de la muestra $(24,6 \%)$ y su puntuación media en la ESO es ligeramente negativa.

Un $16 \%$ de la muestra presenta un patrón de respuesta caracterizado por un bajo grado de confianza en general; son los desconfiados. Podría decirse que es el conglomerado opuesto al primero o de los confiados. Muestran desconfianza hacia categorías concretas, ya sea de personas (en menor grado), ya sea -sobre todo y en mayor medida que el resto de los grupos- de categorías profesionales, con la única excepción del grado de confianza en los científicos, que es algo superior a la del grupo de confiados en las personas de proximidad. Su puntuación en la ESO es positiva, lo que subraya lo destacado más arriba, es decir, la relación entre cierta desconfianza y cierta seguridad: se desconfía desde la seguridad o la confianza en uno mismo.

Por último, un cuarto conglomerado se obtiene a partir de quienes muestran relativa mayor confianza en las categorías personales y, a la vez, relativo bajo grado de confianza con respecto la mayor parte de las categorías profesionales; son los confiados de proximidad. Muestran un grado relativamente alto de confianza en sus vecinos $(5,80) \mathrm{y}$, entre las categorías profesionales, destaca su grado relativamente elevado de confianza en las categorías profesionales que pueden considerarse asimismo de proximidad: en sacerdotes $(5,74)$ o en policías $(6,82)$. Suponen el $23,5 \%$ de la muestra y su puntuación en la ESO es positiva, apuntando cierto grado de seguridad ontológica bajo cierto sentimiento de pertenencia a la comunidad, como mostró Walklate (1998).

La reflexión abierta por la falta de correlación inicial entre el grado de confianza en distintas categorías y la ESO nos conduce a la recuperación de la afirmación de que la confianza en los otros deriva de una necesidad. Pues bien, yendo un poco más allá de los conglomerados construidos y de cómo se agrupan alrededor de las medias en los grados de confianza mostrados hacia distintas categorías, cabe establecer la hipótesis de que los que muestran menos seguridad son los que más necesitan confiar. Así, si se considera el grado de confianza mostrado como el grado de necesidad de confiar, especialmente en sus valores más altos de grado de confianza (10, en nuestra escala), se tendría que los sujetos que de tal manera han contestado tendrían puntuaciones negativas en la ESO. Pues bien, es lo que se observa en la tabla 5, construida a partir de las puntuaciones medias en la ESO de quienes han mostrado el mayor grado de confianza en cada una 
de las categorías (un 10 en la escala de confianza). Tal relación entre declaración de la máxima confianza a la categoría respectiva y puntuación negativa en la ESO tiene en el caso de periodistas $(-0,53)$, ecologistas $(-0,58)$ y psicólogos $(-0,53)$ tres manifestaciones extremas, siendo especialmente llamativa la última para el establecimiento de esa relación entre inseguridad ontológica y dependencia-confianza. Es decir, quienes menos seguridad ontológica presentan están entre los que más necesitan del apoyo psicológico y, por lo tanto, los que más necesitan confiar en estos profesionales; lo que se traduce en una señalización de mayor confianza hacia ellos.

Tabla 5: Puntuaciones en la ESO entre los que tienen el mayor grado de confianza (10) en las distintas categorías

\begin{tabular}{|l|c|c|c|}
\hline Categorías en las que confiar & Media & N & Desviación estándar \\
\hline Personas que acaba de conocer &,- 4455 & 82 & 1,349 \\
\hline Sus vecinos &,- 4946 & 241 & 1,077 \\
\hline Sus amigos &,- 1539 & 727 & 1,044 \\
\hline Sus compañeros de trabajo o estudio &,- 1420 & 182 & 1,049 \\
\hline Familiares directos &,- 1087 & 1460 & 1,005 \\
\hline Familia segundo &,- 2287 & 1121 & 0,988 \\
\hline Los científicos &,- 3121 & 706 & 1,004 \\
\hline Los sacerdotes &,- 2477 & 128 & 1,095 \\
\hline Los médicos &,- 3243 & 550 & 1,039 \\
\hline Los periodistas &,- 5828 & 103 & 1,046 \\
\hline Los psicólogos &,- 5353 & 230 & 1,062 \\
\hline Los jueces &,- 3163 & 60 & 1,245 \\
\hline Los maestros y profesores &,- 3951 & 441 & 0,998 \\
\hline Los ecologistas &,- 5699 & 255 & 0,961 \\
\hline Los bomberos &,- 3499 & 813 & 0,978 \\
\hline Los empresarios &,- 2429 & 56 & 1,058 \\
\hline Los policías &,- 4702 & 312 & 0,998 \\
\hline
\end{tabular}

Fuente: Elaboración propia a partir de la encuesta realizada. 


\section{Las diferencias.}

Con un carácter más exploratorio que analítico-descriptivo, se ha llevado a cabo un análisis de regresión en el que la variable dependiente es la ESO. Como muestra la tabla 6 , hay variables que adquieren cierto peso y significación estadística a la hora de la variabilidad de esta escala. Entre ellas, destaca el peso del género, señalando que el ser varón está relacionado con puntuaciones medias positivas en la ESO, y ser mujer con puntuaciones medias negativas, estando distanciados por un intervalo de 0,53 puntos en la escala.

La edad parece determinar escasamente y de manera poco significativa la ESO. De hecho, solo disminuye en tres milésimas por cada año que se cumple. Eso sí, nos indica que, cuanta más edad, menos sentimiento de seguridad. No obstante, analizando las puntuaciones medias año a año pueden observarse puntuaciones negativas a los 28 y 34 años (probablemente determinadas por otras circunstancias de los individuos encuestados), aunque éstas suelen ser más intensas y generalizadas a partir de los 59 años.

Tabla 6: Análisis de regresión lineal, con variable dependiente Escala de Seguridad Ontológica (coeficientes beta) ${ }^{\mathrm{a}}$

\begin{tabular}{|l|c|}
\hline Edad & $-0,052$ \\
\hline Varón & $0,226^{* * *}$ \\
\hline Nivel bajo estudios & $-0,129^{* * *}$ \\
\hline Universitario & $0,105^{* * *}$ \\
\hline Más de 3000 & $0,084^{* * *}$ \\
\hline Menos de 1200 & $-0,124^{* * *}$ \\
\hline Hijos & $-0,075^{* * *}$ \\
\hline Solo & $0,067^{* * *}$ \\
\hline Solo con hijos & 0,025 \\
\hline Pareja & 0,046 \\
\hline Urbano & $-0,018$ \\
\hline Ocupación & $-0,047^{* *}$ \\
\hline
\end{tabular}

Fuente: Elaboración propia a partir de la encuesta realizada.

${ }^{*} \mathrm{p}<0,100 ; * * \mathrm{p}<0,050 ; * * * \mathrm{p}<0,010$

${ }^{a}$ En el caso de las variables que no son de intervalo, se construyen variables ficticias.

La posición socioeconómica, marcada por el nivel de ingresos en el hogar y el nivel de estudios, aparecen significativamente relacionados con las variaciones en la ESO. A mayor nivel de ingresos y de estudios, mayor es la seguridad ontológica, y, al contrario, las puntuaciones se tornan negativas cuando se desciende en niveles de ingreso y de 
estudios. Así, con las precauciones que conlleva una sustancial disminución de los tamaños submuestrales y de la mediación de la edad que siempre arrastra la variable estudios, mientras que entre quienes viven en hogares con ingresos mensuales medios inferiores a los 3.000 euros y tienen estudios por debajo del Bachiller Superior/BUP las puntuaciones son negativas, entre quienes residen en hogares por encima de esos ingresos, la puntuación es positiva a partir de la posesión del título de Bachiller Elemental/EGB. A su vez, entre los que tienen Bachiller Superior/BUP, la puntuación es positiva a partir de unos ingresos mensuales medios de 1.200 euros y desde cualquier experiencia universitaria -aunque sea sin completar una titulación- entre los que viven en hogares por encima de los 3.000 euros.

La presencia de hijos, como aparecía en el estudio cualitativo, tiene su proyección en la ESO. Entre los que no tienen hijos la puntuación es positiva, mientras entre quienes los tienen la preocupación por su futuro les lleva a puntuaciones negativas $(-0,11)$. Más allá de la presencia de hijos y neutralizando esta variable y la edad al formar parte del modelo, las formas de convivencia apuntan una puntuación ligeramente positiva de quienes viven solos. Analizando las medias y, por lo tanto, sin neutralización alguna, se recoge una puntuación pequeña, cercana a cero $(0,03)$, siendo incluso mayor entre quienes viven con los padres $(0,13)$. Entre quienes viven solos con los hijos y a pesar del coeficiente beta que aparece en la tabla 6 , la puntuación media es negativa $(-0,24)$.

Se pone también de relieve el poco peso del tamaño del hábitat. Su contribución a la variación de la escala de una de las categorías, hábitat urbano (más de 500.000 habitantes) es más pequeña y ligeramente negativa, con escasa significación estadística. No obstante, la puntuación media directa es ligeramente positiva para los tamaños de hábitat por encima de los 50.000 habitantes $(0,08)$ y negativa en los de menor tamaño $(-0,19$, en los que están por debajo de los 10.000 habitantes).

En el comportamiento de la ocupación en la ESO se encuentra uno de los aspectos más interesantes. En el modelo de la tabla 6, aparece con una relación negativa. No obstante, la puntuación media de los ocupados es ligeramente positiva $(0,098)$, aunque muy por debajo de la presentada por los estudiantes $(0,4)$. Si observamos que quienes señalan como actividad el trabajo doméstico no remunerado obtienen la puntuación media negativa más elevada $(-0,49)$, se apunta directamente al importante papel que tiene la variable género. Así, en los varones ocupados, la puntuación media es positiva $(0,25)$, mientras que entre las mujeres, con aproximadamente dos tercios ocupadas, es negativa $(-0,12)$.

Siguiendo con la variable ocupación, la incursión en la misma desde la ESO muestra las notables diferencias que abarca. Así, mientras que entre los asalariados que piensan que es muy probable o bastante probable que durante los próximos doce meses pierdan su empleo actual las puntuaciones medias negativas alcanzan cifras de $-0,29$ (muy probable) y $-0,01$ (bastante probable), entre los que piensan que es poco o nada probable esa pérdida de empleo, se tornan puntuaciones positivas de 0,16 y 0,12 respectivamente. Ello constituye una muestra de la relevancia de las condiciones de seguridad en el empleo para la obtención de un sentimiento de seguridad general en la vida, bastante más allá del ámbito laboral. 
Tabla 7: Regresión logística del hecho de disponer de seguridad ontológica: exponenciación del coeficiente B para cada predictor (odds ratio)

\begin{tabular}{|c|c|c|c|c|c|}
\hline \multirow{2}{*}{ Sexo } & \multicolumn{2}{|l|}{ Varón } & \multirow{8}{*}{ Nivel de estudios } & \multicolumn{2}{|l|}{ Sin estudios } \\
\hline & Mujer & $0,518 * * *$ & & Primarios & 2,507 \\
\hline \multirow{4}{*}{ Edad } & \multicolumn{2}{|l|}{$18-30$} & & $\mathrm{BE} / \mathrm{EGB}$ & $2,882 * *$ \\
\hline & $31-45$ & 0,920 & & $\begin{array}{l}\mathrm{BS} / \mathrm{BUP} \\
/ \mathrm{FP}\end{array}$ & $3,288 * *$ \\
\hline & $46-65$ & 0,764 & & $\begin{array}{l}\text { Uni } \\
\text { incompleto }\end{array}$ & $3,855 * * *$ \\
\hline & Más de 65 & 1,290 & & Uni medio & $6,299 * * *$ \\
\hline \multirow{4}{*}{ Tamaño habitat } & \multicolumn{2}{|c|}{ Menos de 10000 habitantes } & & Uni superior & $5,450 * * *$ \\
\hline & $\begin{array}{l}10.001 \text { a } 50 \\
\text { mil habitantes }\end{array}$ & 1,024 & & Post - Grado & $6,298 * * *$ \\
\hline & $\begin{array}{l}50.001 \text { a } 500 \\
\text { mil habitantes }\end{array}$ & $1,336^{* *}$ & \multirow{3}{*}{ Vivir separacion } & \multicolumn{2}{|l|}{ Sí } \\
\hline & $\begin{array}{l}\text { Más de } 500 \\
\text { mil habitantes }\end{array}$ & 1,109 & & No & $1,376^{* *}$ \\
\hline \multirow{8}{*}{ Actividad } & \multicolumn{2}{|l|}{ Trabaja } & & $\mathrm{NC}$ & 0,699 \\
\hline & Jubilado/a & 0,753 & \multirow{2}{*}{$\begin{array}{l}\text { Sufrido } \\
\text { enfermedad }\end{array}$} & \multicolumn{2}{|l|}{ Sí } \\
\hline & Pensionista & $0,410 * *$ & & No & 0,954 \\
\hline & Parado/a & 0,999 & \multirow{8}{*}{ Ideología } & \multicolumn{2}{|l|}{1 Izquierda } \\
\hline & $\begin{array}{l}\text { Busca su } \\
\text { primer empleo }\end{array}$ & 0,888 & & 2 & 1,170 \\
\hline & Estudiante & $1,421 * *$ & & 3 & $1,618 * *$ \\
\hline & $\begin{array}{l}\text { Trabajo } \\
\text { doméstico }\end{array}$ & 0,932 & & 4 & $1,434 * *$ \\
\hline & Otra situación & 0,502 & & 5 & 1,043 \\
\hline \multirow{3}{*}{ Hijos } & \multicolumn{2}{|l|}{ Sí } & & 6 & $1,539^{*}$ \\
\hline & No & 1,236 & & 7 & 1,202 \\
\hline & $\mathrm{NC}$ & 0,522 & & 8 & 0,845 \\
\hline
\end{tabular}




\begin{tabular}{|c|c|c|c|c|c|}
\hline \multirow{6}{*}{$\begin{array}{l}\text { Formas de } \\
\text { convivencia }\end{array}$} & \multicolumn{2}{|l|}{ Solo/a } & \multirow{4}{*}{ Ideología } & 9 & 0,673 \\
\hline & $\begin{array}{l}\text { Solo/a con su/s } \\
\text { hijo/a/s }\end{array}$ & 0,702 & & 10 Derecha & $0,629^{*}$ \\
\hline & $\begin{array}{l}\text { Con su marido } \\
\text { o mujer o } \\
\text { pareja }\end{array}$ & 0,825 & & NS & 0,830 \\
\hline & $\begin{array}{l}\text { Con su padre } \\
\text { y/o madre con } \\
\text { o sin hermanos }\end{array}$ & $0,651 * * *$ & & $\mathrm{NC}$ & 0,683 \\
\hline & Otra situación & 0,822 & \multirow{5}{*}{ Religíon } & Católico/a & \\
\hline & N.C. & 3,875 & & $\begin{array}{l}\text { Creyente de } \\
\text { otra religión }\end{array}$ & $1,620 * *$ \\
\hline \multirow{4}{*}{ Nivel de ingresos } & \multicolumn{2}{|l|}{ Menos de 500} & & No creyente & $1,393 * *$ \\
\hline & $\begin{array}{l}\text { Entre } \\
501 \text { y } 1200\end{array}$ & 1,129 & & Ateo/a & $1,759 * * *$ \\
\hline & $\begin{array}{l}\text { Entre } \\
1201 \text { y } 3000\end{array}$ & $1,610^{* *}$ & & N.C. & $2,241 * * *$ \\
\hline & $\begin{array}{l}\text { Más } \\
\text { de } 3000 \text { eur }\end{array}$ & $2,781 * * *$ & \multicolumn{3}{|c|}{ El pseudo $\mathrm{R}^{2}$ (Nagelkerke) es 0,218} \\
\hline
\end{tabular}

Con subrayado en gris: situación de referencia ${ }^{*} \mathrm{p}<0,100 ; * * \mathrm{p}<0,050 ; * * * \mathrm{p}<0,010$

Fuente: Elaboración propia a partir de la encuesta realizada. 


\section{Gráfico 1: Efectos variables sociodemográficas sobre probabilidad disponer seguridad ontológica}

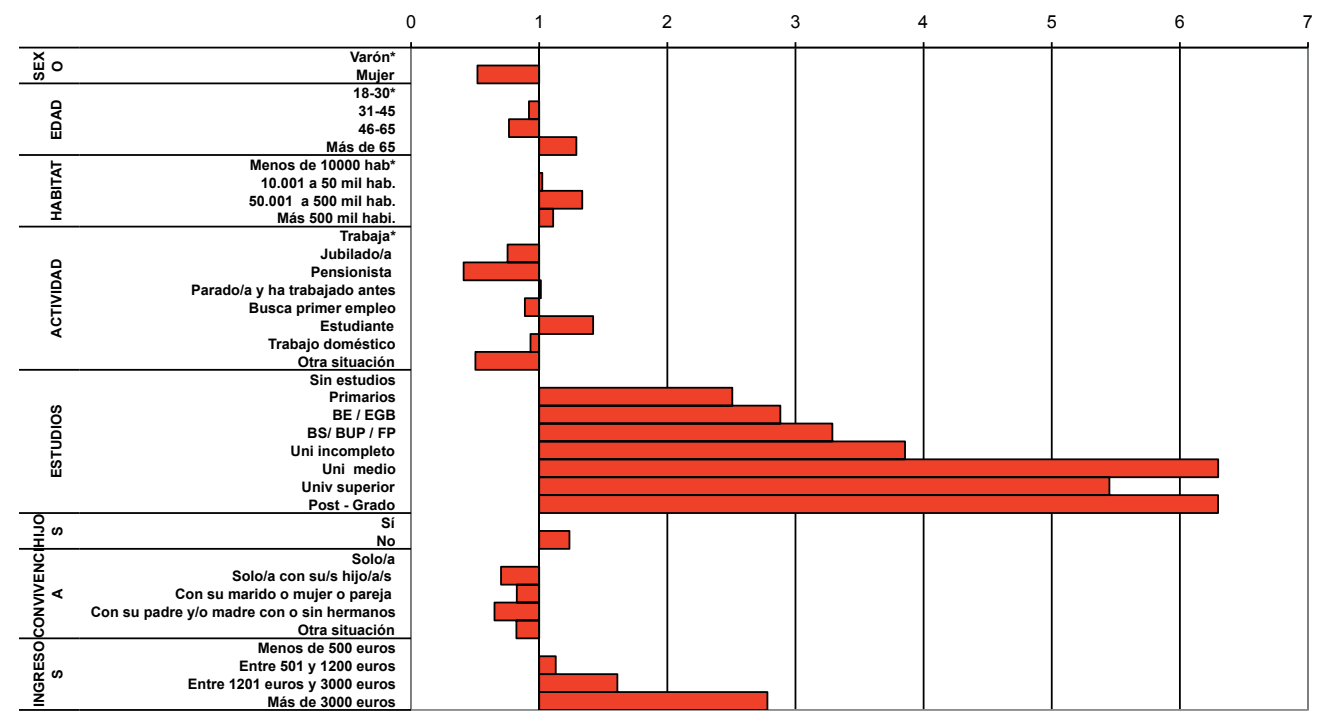

*Situación de referencia

Los resultados han sido obtenidos mediante regresión logística del hecho de tener seguridad ontológica (puntuación positiva en la ESO)

Lectura: entre una persona que vive en un hogar de con ingresos mensuales por debajo de los 600 euros (situación de referencia) y una persona que vive en un hogar con ingresos mensuales por encima de los 3000 euros, la probabilidad de disponer de seguridad ontológica (odds ratio) se multiplica por 2,8.

Fuente: Elaboración propia a partir de la encuesta realizada. 
Gráfico 2: Efecto variables experienciales sobre probabilidad de disponer seguridad ontológica

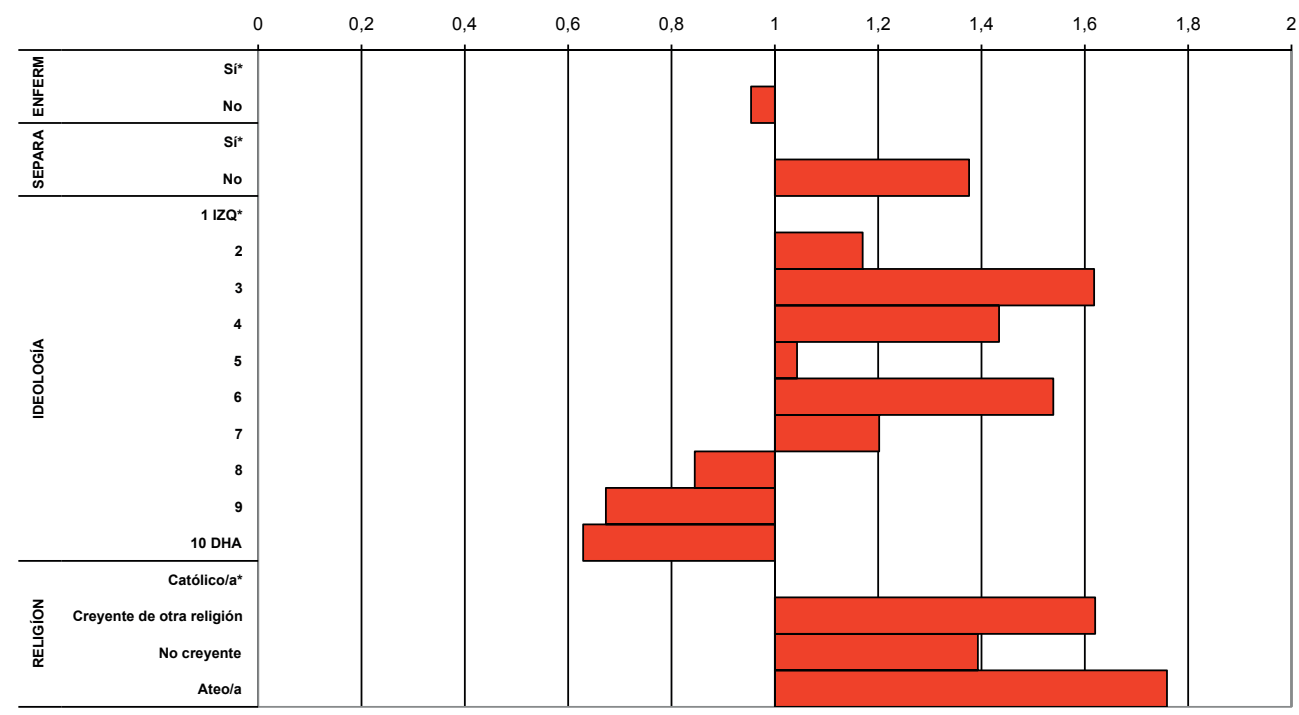

*Situación de referencia

Los resultados han sido obtenidos mediante regresión logística del hecho de tener seguridad ontológica (puntuación positiva en la ESO)

Lectura: entre una persona que se define como católico (situación de referencia) y una persona que se define como no creyente, la probabilidad de disponer de seguridad ontológica (odds ratio) se multiplica por 1,4 .

Fuente: Elaboración propia a partir de la encuesta realizada.

\section{Conclusiones}

La profundización en una investigación sobre riesgo, incertidumbre y vulnerabilidad, en el contexto de la crisis económica que afecta a España, se topa con la cuestión sobre cómo recoger sentimientos de seguridad/inseguridad, qué grado alcanzan y en qué medida las distintas posiciones en la estructura social tienen una relación distinta con ellos. Para ello, se parte de que ese sentimiento atraviesa el conjunto de la vida de los individuos, yendo más allá de una única fuente causal, y de la necesidad de crear un instrumento para su observación empírica, lo que se hace a partir del material aportado por unos grupos de discusión y los resultados de una encuesta con cuestionario aplicada telefónicamente a una muestra nacional. 
Los discursos espontáneos producidos por los grupos de discusión ponen en un primer plano y con relativa independencia de la distinta fuente/grado de riesgo a que están expuestos (que sirvió para el diseño de los GDs), sentimientos generalizados de temor e inseguridad, como una especie de telón de fondo de inseguridad. Es también en el material cualitativo donde aparecen algunos elementos que apuntan a distintos grados de inseguridad que, yendo más allá de la distancia con la fuente específica de riesgo, se relacionan con condiciones vitales de los sujetos $\mathrm{y}$, por lo tanto, con categorías sociales diferenciadas. Así, desciende el sentimiento de seguridad cuando median los hijos o la incertidumbre en el empleo.

Siendo el reto abordado por este trabajo la construcción de una instrumento capaz de dar cuenta del concepto seguridad ontológica, de manera que su utilidad fundamental estuviese en articular distintas fuentes de riesgo suponiendo que hay un sentimiento común con relación a ellas y que, por otro lado, se pudiesen observar las diferencias con respecto a ese sentimiento general de las distintas categorías sociales, se hacía preciso acudir a una práctica de investigación distributiva que, a la vez, apuntara indirectamente a ese concepto. La elaboración de una escala (Escala de Seguridad Ontológica: ESO) a partir de preguntas indirectas sobre preocupaciones y sentimientos de seguridad/inseguridad -sin preguntar directa y públicamente sobre un sentimiento de seguridad, sobre el que existe una fuerte mediación cultural en su presentación públicaresultó ser lo más pertinente, aun cuando hay que reconocer que el nudo entre emoción y racionalización está siempre presente.

Con relación a la confianza, que es uno de los objetos de la investigación matriz, laESO genera una observación aparentemente contradictoria. Por un lado, cuando se aborda la confianza en categorías sociales concretas, ya sean relacionales o profesionales (sistema experto), domina la correlación negativa, de manera que la seguridad ontológica estaría vinculada a una falta de confianza en las categorías y, proyectando los resultados, en las instituciones. Sin embargo, aparece positivamente relacionada con expresiones de confianza generalizada. Es decir, el sentimiento de seguridad permite no tener miedo de tratar con la gente, pudiéndose admitir como básico para la existencia de cierto cemento social, abriendo la posibilidad de las relaciones sociales. Por lo tanto, existe una relación entre confianza y seguridad más compleja que la apuntada por Giddens y que cabe abordar en futuros trabajos. En éste, se muestra la relación entre seguridad ontológica y quienes se muestran generalmente desconfiados. Por otro lado, los que manifiestan confianza en los próximos (familia, amigos, vecinos), es decir, el mundo de la vida cotidiana en términos de Schutz, muestran un sentimiento de seguridad. De hecho, en el estudio cualitativo, la casa de los padres aparece como una especie de fuente-refugio matriz de seguridad básica.

Ahora bien, los confiados de forma generalizada y los que dan preferencia a la confianza en los expertos aparecen vinculados a puntuaciones negativas en la ESO, en convergencia con la concepción, principalmente luhmaniana (Luhmann, 1996), de que se confía por necesidad. La situación que genera cierto sentimiento de inseguridad produce necesidad de confianza.

Las aportaciones sustanciales de este trabajo suponen una aproximación a un sentimiento articulado de los temores y preocupaciones de los ciudadanos, resumido 
en el concepto de seguridad ontológica, con relativa independencia de cuáles sean las fuentes específicas que los alimentan. Asimismo, se apunta el grado en que tal sentimiento de seguridad o inseguridad está relacionado con las características sociológicas y de posición en la estructura social de los individuos, de manera que -dejando entre paréntesis las diversas estructuras de personalidad- hay perfiles sociológicos más inclinados al sentimiento de seguridad -varón, nivel de ingresos altos, estudios superiores y actividad laboral- que otros.

Todos estos resultados han sido obtenidos a partir de una escala construida a través de un análisis factorial. Se ha utilizado directamente la puntuación factorial resultante en la matriz factorial, apuntándose la presencia de una dimensión latente. No obstante, se requeriría una futura elaboración para su generalización y potencial uso en nuevos estudios.

\section{Bibliografía}

Berenzin, M. (2009): "Exploring emotions and the economy: new contributions from sociological theory", Theory and Society, 38, pp.335-346.

Barbalet, J. (1998): Emotion, Social Theory, and Social Structure: A Macrosociological Approach, Cambridge, UK: Cambridge University Press.

Berenskoetter, F. y B. Giegerich (2010): "From NATO to EDIP: A Social Constructivist Analysis of German Strategic Adjustment alter the End of the Cold War", Security Studies, 19 (3), pp. 407-452.

Bericat, E. (2000): “La sociología de la emoción y la emoción en la sociología”, Papers. Revista de Sociología, 62, pp. 145-176.

Bericat, E. (2012): "Emociones", Sociopedia.isa, ISA (www.isa-sociology.org/publ/sociopedia-isa).

Bolívar, I.J. (2006): Discursos emocionales y experiencias de la política, Bogotá, Universidad de los Andes.

Bondi, L. (2014): "Feeling insecure: a personal account in a psychoanalytic voice", Social \& Cultural Geography, 15 (3), pp. 332-250.

Brader, T, G.E. Marcus y K.L. Miller (2011): "Emotions and Public Opinion”, en G.C. Edwards III, L.R. Jacobs y R.V. Shapiro (eds.), The Oxford Handbook of American Public Opinion and the Media. Disponible en:

http://www.oxfordhandbooks.com/ doi: 101093/oxfordhb/9780199545636.003.0024

Burges, J.P. (2012): "Value, security and temporality in Nietzsche's critique of modernity", The Sociological Review, 60 (4), pp. 696-714.

Broom, A. y E. Kirby (2013): "The end of life and the family: hospice patients' views on dying as relational", Sociology of Health and Illness, 35 (4), pp. 499-513.

Castel, R. (2009) La montée des incertitudes, Paris, Seuil.

Cohen, J. y M. Metzger (1998) "social affiliation and the achievement of ontological security through interpersonal and mass communication", Critical Studies in Mass Communication, 15 (1), pp. 41-60. 
Collins Dictionaries (2014): Collins English Dictionary: Complete and Unabridged (12 $2^{\text {th }}$ edition), Harper-Collins, Nueva York.

Delehanty, D.K. y B.J. Steele (2009): "Engaging the narrative in ontological (in) security: insights from feminist IR", Cambridge Review of International Affairs, 22 (3), pp. 525-540.

Diener, E., J.F. Helliwell y D. Kahneman (eds.) (2010): Differences in Well-Being, Nueva York, Oxford University Press.

Douglas, M. y A. Waldavsky (1982): Risk and Culture: An Essay on the Selection of Technical and Environmental Dangers, Berkeley y Londres, Univ. of California Press.

Dupuis, A. y D.C. Thorns (1998): "Home, Home Owenership and the search for Ontological Security", Sociological Review, 46 (1), pp. 24-47.

Ekman, P. (1982): Emotion in the Human Face, Cambridge, Cambridge University Press.

Elias, N. (1987): El proceso de civilización, Madrid, Fondo Cultura Económica.

Emirbayer, M. y Ch.A. Goldberg (2005): "Pragmatism, Bourdieu, and collective emotions in contentious politics", Theory and Society, 34 (5-6), pp. 469-518.

Featherstone, M. (1991): Consumer culture and Postmodernism, Londres, Sage.

Georgiou, M. (2013): "Seeking Ontological Security beyond the Nation: The Role of Transnational Television", Television \& New Media, 14 (4), pp. 304-321.

Giddens, A. (1984): The Constitution of Society, Cambridge, Polity Press.

Giddens, A. (1990): Consequences of Modernity, Standford, Standford Universtiy Press.

Giddens, A. (1991): Modernity and Self-identity: Self and Society in the Late Modern Age, Standford, Standford Universtiy Press.

Giddens, A. (1992): Transformaciones de la intimidad, Madrid, Cátedra.

Girling, J. (2006): Emotion and Reason in Social Change, Nueva York, Palgrave.

Gordon, S. L. (1981): "The Sociology of Sentiments and Emotion", en M. Rosenberg y R. H. Turner (eds.), Social Psychology: Sociological Perspectives, New York: Basic Books, pp. 562-592.

Gordon, S. L. (1989): "Institutional and Impulsive Orientations in Selectively Appropriating Emotions to Self" en D. D. Franks y E. D. McCarthy (eds.), The Sociology of Emotions: Original Essays and Research Papers, Greenwich, CT: JAI Press, pp. 115-135.

Gustaffson, K. (2014): "Memory Politics and Ontological Security in Sino-Japanese Relations", Asian Studies Review, 38 (19), pp. 71-86.

Hair, J.F., R.E. Anderson, R.L. Tatham y W.C. Black (1999): Análisis multivariante, Madrid, Prentice-Hall.

Harney, N.D. (2012): "Migrant strategies, informal economies and ontological security: Ukrainians in Naples, Italy", International Journal of Sociology and Social Policy, 32 (1-2), pp. 4-16.

Harries, T. (2008): "Feeling Secure or being secure? Whay it can sem. Better not to Project yourseflf against a natural hazard", Health, Risk \& Society, 10 (5), pp.479490. 
Hawkins, R.L. y K. Maurer (2011): “'You fix my community, you have fixed my life': the disruption and rebuilding of ontological security in New Orleans", Disasters, 35 (1), pp.143-159).

Hinton, D.E. (2009): "Nightmares Among Cambodian Refugees: The Breaching of Concentric Ontological Security", Culture, Medicine and Psychiatry, 33 (2), pp. 219-265.

Hochschild, A. R. (1979): "Emotion Work, Feeling Rules, and Social Structure", American Journal of Sociology 85, pp. 551-575.

Hochschild, A. R. (1983): The Managed Heart: Commercialization of Human Feeling, Berkeley, University of California Press.

Hochschild, A. R. (1989): The Second Shift: Working Parents and the Revolution at Home. New York, Viking Press.

Jaspers, J.M. (2011): "Emotions and Social Movements: Twenty Years of Theory and Research", Annual Review of Sociology, 37, pp. 285-303.

Kemper, D. L. (ed.) (1990): Research Agendas in the Sociology of Emotions, Albany: State University of New York Press.

Kinnvall, Catarina (2004): "Globalization and Religious Nationalism: Self, Identity, and the Search for Ontological Security", Political Psychology, 25 (5), 741-767.

Kury, H. (1996): "Desarrollo de la delincuencia en Europa oriental y occidental. Una comparación entre distintos países", Revista de Derecho Penal y Criminología, 6, pp.599-687.

Le Bon, G. (2000): Psicología de las masas, Madrid, Morata.

Lemmings, D. y A. Brooks (2014): Emotions and Social Change. Historical and Sociological Perspectives, Nueva York, Routledge.

Lukacs, G. (2007): Marx, ontología del ser social, Madrid, Akal.

Luhmann, N. (1996): Confianza, Barcelona, Anthropos.

Marks, N. (2011): The Happiness Manifesto: How Nations and People Can Nurture Well-Being, Nueva York, TED Books.

Maturana, H. (2001): Emociones y lenguaje en educación y política, Santiago, Dolmen.

Mitzen, J. (2006): "Ontological Security in World Politics: State Identity and the Security Dilemma", European Journal of International Relations, 12 (3), pp. 341370

Newton, J. (2008): "Emotional attachment to home and security for permanent residents in caravan parks in Melbourne", Journal of Sociology, 44 (3), pp.219-232.

Ostertag, F.N. (2010): "Processing Culture: Cognition, Ontology, and the News Media', Sociological Forum, 25 (4), pp. 824-850.

Pretorius, R. (2008): "Armed Robbery, Violent Assault and Perceptions of Personal Insecurity and Society as a Risk", Acta Criminologica, 21 (2), pp. 81-91.

Rajah, V. (2010): "Intimacy, Time, and Scarcity: Drug-Involved Women Account for Secretly Withholding Financial Capital in Violent Intimate Relationships", Journal of Contemporary Ethnography, 39 (2), pp. 131-158.

Roe, P. (2014): “Gender and 'positive' security", International Relations, 28 (1), pp. 116-138. 
Ross, L.M. y G. Squires (2011): "The Personal Costs of Subprime Lending and the Foreclosure Crisis: A Matter of Trust, Insecurity, and Institutional Deception", Social Science 4, 92 (1), pp.140-163.

Scheff, T.J. (1990): Microsociology: Discourse, Emotion and Social Structure, Chicago y Londres, University of Chicago Press.

Schutz, A. (1974a): Estudios sobre teoría social, Buenos Aires, Amorrortu.

Schutz, A. (1974b): El problema de la realidad social, Buenos Aires, Amorrortu.

Silverstone, R. (1993): "Television, Ontological Security and the Transitional Object", Media, Culture \& Society, 15 (4), pp. 573-598.

Silverstone, R. (2006): Media \& Morality: On the Rise of the Mediapolis, Cambridge, Polity Press.

Smelser, N.J. (1998): "The Rational and the Ambivalent in the Social Sciences", American Journal of Sociology, 63, pp.1-15.

Steele, B. (2005): "Ontological Security and the Power of Self-Identity: British Neutrality and the American Civil War", Review of International Studies, 31 (3), pp. 519-540.

Tarde, G. (1989 [e.o. 1901]): L'opinion et la foule, París, Les Presses universitaires de France.

Taylor, S (2013): "Searching for ontological security: Changing meaning of home amongst a Punjabi diaspora", Contributions to Indian Sociology, 47 (3), pp. 395422.

Turner, J.H. (1999): “Toward a general sociological theory of emotions”, Journal for the Theory of Social Behaviour, 29 (2), pp. 133-162.

Turner, J.H. y J.E. Stests (2005): The Sociology of Emotions, Nueva York, Cambridge University Press.

Vornanen, R., M. Torronen y P. Niemela (2009): "Insecurity of young people”, Young, 17 (4), pp.399-419.

Wharton, A.S. (2009): "The sociology of emotional labor", Annual Review of Sociology 35 , pp. 147-165.

Walklate, S. (1998): “Crime and community: fear or trust?", British Journal of Sociology, 49 (4), pp.550-570.

Walklate, S. (2006): "Community safety and victims: who is the victim of community safety?" en P. Squires (ed.), Community Safety: Critical Perspectives on Policy and Practice, Bristol, Policy Press.

Williams, R. (2011): “'This Is the Night TV Died': Television Post-Object Fandom and the Demise of the West Wing", Popular Communication, 9 (4), pp.266-279.

Zimbardo, Ph.G. y J.N. Boyd (1999): "Putting time in perspective: A valid, reliable individual-differences metric" Journal of Personality and Social Psychology, 77 (6), pp. 1271-1288. 